\title{
HIERARCHICAL METHOD FOR NURSE ROSTERING BASED ON GRANULAR PRE-PROCESSING OF CONSTRAINTS
}

\author{
Geetha Baskaran \\ School of Foundation studies \\ University of Nottingham, Malaysia Campus \\ Jalan Broga , 43500 Semenyih, Selangor. \\ E-mail: Geetha.Baskaran@nottingham.edu.my
}

\author{
Andrzej Bargiela \\ School of Computer Science \\ University of Nottingham, Malaysia Campus \\ Jalan Broga , 43500 Semenyih, Selangor. \\ E-mail: abb@cs.nott.ac.uk
}

\author{
Rong Qu \\ School of Computer Science \\ University of Nottingham, Jubilee Campus \\ Nottingham NG8 1BB, UK. \\ E-mail: rxq@cs.nott.ac.uk
}

\section{KEYWORDS}

Hierarchical Method, Nurse Rostering, Scheduling, Granulation, Simulation.

\begin{abstract}
Nurse Rostering problems represent a subclass of scheduling problems that are hard to solve. Their complexity is due to the large solution spaces and the many objectives and constraints that need to be fulfilled. In this study, we propose a hierarchical method of granulation of problem domain through preprocessing of constraints. A set of zero-cost patterns in the granulated search space provides a basis for the generation of work schedules. Feasible schedules calculated for week 1 are used to define zero-cost shift patterns that can be deployed in week 2 . These in turn are used for the generation of feasible schedules for week 2. The process can be applied over the extended time frame. We show that the granulation of the problem description in terms of constraints and scheduling time frames leads to a more manageable computing task.
\end{abstract}

\section{INTRODUCTION}

In recent years, many hospitals have moved to selfscheduling where nurses create their own rosters based on eligibility and contractual agreements. When conflicts occur, adjustments are made through consensus. Although it is easy to implement, selfscheduling may not be practical in all situations. As a consequence, more systematic approaches have been developed (Hadi and Jonathan, 2007).

Nurse rostering is concerned with highly constrained resource allocation problem in to slots in a pattern, where the pattern denotes a set of legal shifts. In nurse rostering, the ultimate aim is to create high quality timetables, considering the well-being of nurses and the operational concerns of the employers (Ozcan 2005). Typical issues concerned with nurse rostering include coverage demand, day-off requirements, weekend-off requirements, minimum and maximum workforce, etc (Brucker et al. 2005).

A wide variety of different methodologies and models have been developed to deal with different problem circumstances during the years (Cheang et al. 2003, Ernst et al. 2004, Sitompul and Randhawa, 1990). These include mathematical programming (Beaumont, 1997, Pierskalla and Rath , 1976, Warner, 1976), meta-heuristic methods such as Genetic Algorithms (GA), Constructive Heuristic, Simulated Annealing, Tabu-Search, Multi Objective Approach (Aickelin and Dowsland , 2003, Burke et al.,2001, Burke et al., 1998, Duenas et al., 2003, Gendrau et al., 2003) and constraint satisfaction techniques (Adbennadher and Schlenker,1999, Berrada et al., 1996, Meyer and Hofe, 2000, Nonobe and Ibaraki, 1998). Column generation was employed in nurse scheduling (Bard and Purnomo, 2005) using set covering-type models and integer programming. Knowledge based techniques have also been explored for solving nurse rostering problems (Beddoe and Petrovic, 2005, Lukman et al., 1991).

For an in-depth review of the nurse rostering literature, see (Burke et al. 2004). A brief summary follows. Miller et al. (1976) were the first to formally address the preference scheduling problem. Starting with an initial solution, they developed a greedy neighborhood search procedure to find local optima (Randhawa and Sitompul, 1993). More recently, metaheuristics, such as tabu search, have been designed for various nurse rostering problems. The distinguishing feature of these methods is their ability to escape local optima.

Starting with several initial solutions, GAs use crossover and mutation operators to generate pairs of improved solutions. This representation allowed the original problem to be viewed as a permutation problem, which is more suitable for GAs due to the nature of the exchange procedures. The column generation approach, an example of decomposition, uses the pattern-view formulation as a master problem and either heuristics or the shift-view formulation as sub problems to generate nurse rosters. In the vast 
majority of cases, the pattern-view formulation only includes demand constraints, which gives it the advantage of simplicity, although the number of columns grows exponentially with the length of the planning horizon. Alternatively, goal programming is a common methodology for dealing with soft constraints. In this approach, rules are prioritized and treated as goals or objectives to be satisfied. The optimization is carried out sequentially so as to ensure the achievement of the goal.

Constraint programming (CP) is also an $\mathrm{AI}$ technique that, unlike integer programming, does not make use of an explicit mathematical representation of the hard and soft constraints. Instead, the constraints are used to formulate propagation rules that reduce the search space. When constraint satisfaction is difficult to achieve, each constraint is assigned a different priority that is used to drive the search process. Solution quality is judged on the basis of priority satisfaction (Hadi and Jonathan, 2007).

\section{NURSE ROSTERING PROBLEM}

The terms scheduling and rostering are defined (Anthony, 1995) as follows:

- Scheduling is the allocation, subject to constraints, of resources to objects placed in space-time, in such a way as to minimize the total cost of the resources used.

- Rostering is the placing, subject to constraints, of resources into slots in a pattern.

Nurse rostering problem involves allocating the required workload to nurses subject to a number of constraints. Constraints are categorized into two groups; Hard and Soft Constraints, which vary with legal regulations and individual preferences.

- Hard Constraints are those that must be satisfied to obtain feasible solutions.

- Soft Constraints are typically time related constraints. They are desirable but not compulsory, and thus can be violated.

Without the loss of generality we discuss our contribution in the context of a specific nurse rostering problem as encountered by ORTEC in intensive care units in Dutch hospitals. This nurse rostering problem has the following characteristics.

1. Work requirements adhere to Dutch national legislation, and the collective labor agreements in force in Dutch hospitals.

2. The requests of the personnel are considered to be very important, and should be met as much as possible; These are represented as soft constraints.

3. It is not necessary to consider qualifications, as all personnel are highly qualified. Nurses still in training are disregarded in the original planning (and are added to the schedule afterwards).

4. The size of the department is rather small, but convenient for testing purposes.
We focus on creating weekly schedules for a ward with 15 nurses. The problem is to assigning a certain number of different types of shifts to 15 nurses in a ward within a scheduling period of 5 weeks. The required number of nurses on individual shifts for different days of the week is summarised in Table 1. The study is focussed on nurses with full-time contracts of 36 hours per week.

\section{Shifts and personnel cover demand}

There are 4 shift types in the problem. Each shift types covers 9 hours including one hour of resting time, except the night shift, which does not have resting time. So there are 8 actual working hours for each of these shift types.

In designing the shift sequences, we first consider shifts which are the most important or most difficult to schedule. In this problem, night shifts are the most important ones. This will result in three basic shift patterns presented in Table 2. These valid patterns will repeat for week 1 until 5.

Table 1. Shift Types and Demand during a Week

\begin{tabular}{|l|l|l|l|l|l|l|l|l|l|}
\hline Shift & Start & End & \multicolumn{7}{|c|}{ Demand } \\
\cline { 4 - 10 } Type & Time & Time & M & T & \multicolumn{1}{|c|}{ W } & \multicolumn{1}{|c|}{ F } & \multicolumn{1}{c|}{ S } \\
\hline Early & 07.00 & 16.00 & 3 & 3 & 3 & 3 & 3 & 2 & 2 \\
\hline Day & 08.00 & 17.00 & 3 & 3 & 3 & 3 & 3 & 2 & 2 \\
\hline Late & 14.00 & 23.00 & 3 & 3 & 3 & 3 & 3 & 2 & 2 \\
\hline $\begin{array}{l}\text { Nigh } \\
\text { t }\end{array}$ & 23.00 & 07.00 & 1 & 1 & 1 & 1 & 1 & 1 & 1 \\
\hline
\end{tabular}

$$
\text { Night }=\mathrm{N} \quad \text { Early }=\mathrm{E} \quad \text { Day }=\mathrm{D} \quad \text { Late }=\mathrm{L}
$$

Table 2: Basic Night Shift Patterns

\begin{tabular}{|c|c|c|c|c|c|c|c|}
\hline & M & T & W & T & F & S & S \\
\hline Pattern A & N & N & - & - & - & - & - \\
\hline Pattern B & - & - & N & N & - & - & - \\
\hline Pattern C & - & - & - & - & N & N & N \\
\hline
\end{tabular}

The hard and soft constraints that need to be satisfied are described below respectively. Currently, hard constraint 11 and soft constraint such as 3b, 5b and 6b are not included in these studies.

\section{Hard Constraints}

The hard constraints listed below must be met in any circumstances otherwise the schedule is considered to be infeasible and unacceptable.

1) Demands need to be fulfilled

2) For each day, 1 nurse may start only one shift.

3) Within a scheduling period, a nurse is allowed to exceed the number of hours for which he/she is available for his/her department by at most 4 hours.

4) The maximum number of night shifts is 3 per period

of 5 consecutive weeks.

5) A nurse must receive at least 2 weekends off duty 
per 5 week period. A weekend off duty lasts 60 hours including Saturday 00:00 to Monday 04:00.

6) Following a series of at least 2 consecutive night shifts, a 42 hours rest is required.

7) During any period of 24 consecutive hours, at least 11 hours of rest is required. A night shift has to be followed by at least 14 hours rest. An exception is that once in a period of 21 days for 24 consecutive hours, the resting time may be reduced to 8 hours.

8) The number of consecutive night shifts is at most 3.

9) The number of consecutive shifts (workdays) is at most 6 .

10) One of the full-time nurses requires not receiving any late shifts.

11) The maximum labour time per week is on average 36 hours over a period of 13 consecutive weeks if this period does not include work during night shifts.

\section{Soft Constraints}

Soft constraints, in the problem we are dealing with are listed below. Ideally these constraints should be satisfied as much as possible. However, in real world circumstances, it is usually necessary to violate some of these soft constraints. Depending on how strongly these soft constraints are desired (especially in comparison to other soft constraints).

1) For the period of Friday 23:00 to Monday 0:00, a nurse should have either no shifts or at least 2 shifts (Complete Weekend).

2) Avoid sequence of shifts with length of 1 for all nurses.

3a) For nurses with availability of 30-36 hours per week, the length of a series of night shifts should be within the range [2, 3]. It could be part of, but not before, another sequence of shifts.

3b) For nurses with availability of 0-30 hours per week, the length of a series of night shifts should be within the range [2, 3]. It could be part of, but not before, another sequence of shifts.

4) The rest after a series of day, early or late shifts is at least 2 days.

5a) For nurses with availability of 30-36 hours per week, the number of shifts is within the range $[4,5]$ per week.

5b) For nurses with availability of 0-30 hours per week, the number of shifts is within the range [2, 3] per week.

$6 a)$ For nurses with availability of 30-36 hours per week, the length of a series of shifts should be within the range of $[4,6]$.

6b) For nurses with availability of 0-30 hours per week, the length of a series of shifts should be within the range [2, 3].

7) For all nurse, the length of a series of early shifts should be within the range [2, 3]. It could be within another series of shifts.

8) For all nurse the length of a series of late shifts should be within the range of [2, 3]. It could be within another series of shifts.

9a) An early shift after a day shift should be avoided. 9b) An early shift after a late shift should be avoided.

9c) A day shift after a late shift should be avoided.

10) A night shift after an early shift should be avoided.

\section{PROPOSED SOLUTION}

Constraints listed in the previous section are defined at a very detailed time-resolution. In this form they can be quite overwhelming given the number of shifts in the planning horizon and the number of personnel to be scheduled. Following from the observation that there are only three feasible night-shift patterns that satisfy the hard constraints, as listed in Table 2, we proposed to identify feasible day-shifts in a similar way. This in effect represents abstraction from the detailed specification of hard constraints and the development of new semantical entities of feasible day- and nightpatterns to express the nurse-scheduling problem. In the context of Granular Computing we interpret this as granulation of constraints.

From the description of the shifts it is clear that the E, D and L type shifts are similar in terms of working hours and also in terms of work regulations that are applicable to them. By contrast, the night shifts represent a very different entity. We propose therefore that the nurse-scheduling problem may be expressed at a more abstract level using just two types of shifts daytime and night-time. Clearly this granulation of shift types implies introduction of hierarchical processing of schedules, since the final solution needs to be defined in terms of E, D, L and N shifts. Nevertheless, such a partitioning of the problem is advantageous because the large solution space of feasible schedules becomes significantly smaller for just two shift types and the post-processing task from day-time shifts into detailed $\mathrm{E}, \mathrm{D}, \mathrm{L}$ shifts is relatively easy.

Having defined the granular entities of shifts and patterns we proceed to formulate the nurse-scheduling problem as a recursive process with a time frame of one week. We postulate that a granulation of the scheduling horizon from individual days into weeks correlates closely with the granulation of constraints into patterns and consequently provides a natural simplification of the scheduling problem from the full-schedulinghorizon to a recursive-weekly-scheduling. Clearly, the search space that is pertinent to finding a weekly schedule is significantly smaller than the corresponding search space for the full-scheduling-horizon task.

The feasible schedules for week 1 for the granular nurse-scheduling problem can be enumerated relatively easily and can be seen as a new granular search space for the solutions for subsequent weeks. Since these schedules capture the essence of hard constraints and the requirements for personnel cover (which are predefined for the scheduling horizon) this set is exhaustive and can only be reduced by the introduction of additional constraints. As a result of the above granulations, the scheduling task becomes spanned by the relatively small vector of feasible patterns and schedules for week 1 . 
The overall idea outlined above is to reduce the problem complexity by granulating the search space. The algorithm implementing this idea is outlined in the following:

1. Convert the problem space from $\{\mathrm{N}, \mathrm{E}, \mathrm{L}, \mathrm{D}, \mathrm{R}\}$ to a smaller space of $\{\mathrm{N}, \mathrm{d}, \mathrm{R}\}$.

[The result of the granulation of the shift types is the reduction of the number of feasible shift patterns by several orders of magnitude. This point is illustrated in Figure 3. A large number of patterns of the type NNRRDDD, NNRRRDLL, NNRRLLL, etc. are all represented by a single pattern NNRRddd. The saving implied by this granulation of shift types is pronounced even more strongly if one considers several weeks. If the number of detailed patterns represented by a single general pattern is $\mathrm{K}$, then in a 5-week schedule one will have $\mathrm{K}^{5}$ detailed patterns represented by a single general pattern. Notice that Pattern A in Table 2 reflects the same insight in the context of night shifts.]

2. Identify all the shift patterns with " 0 " cost for week 1 in the $\{\mathrm{N} \mathrm{d} \mathrm{R}\}$ space.

[This is carried out as offline processing of all hard and soft constraints. Although some of the hard constraints such as constraint 4 and 5 are specified in the context of 5 weeks scheduling horizon, they can be easily interpreted in the context of a oneweek scheduling with an appropriate additional constraint imposed onto subsequent weeks. The resulting set of 26 shift patterns is listed in Table 4.]

3. Within the space of feasible shift patterns for week 1 we identify sets of patterns that satisfy the personnel cover requirements, as specified in Table 3 , which have cardinality equal to the number of nurses on the ward.

[In the case of our specific ward we have identified some 380 sets with cardinality 15 satisfying the personnel cover requirement of "9 999966 " for day-shifts and "1 11111111 ”" for night-shifts. These 380 sets represent feasible schedules for week 1 . It should be noted that by specifying different cardinality of the sets we can generate feasible schedules for different number of staff. Also we can easily take into account different requirements for personnel cover].

4. Extending the scheduling horizon from week " $w$ " to " $w+1$ " we identify which of the feasible 26 shift patterns need to be excluded for each specific pattern deployed in week "w”.

[This step ensures that the hard constraints on the number of consecutive shifts on the interface of two weeks and the number of night shifts for a single nurse are satisfied. Note that this step reduces the search space of feasible schedules for week " $w+1$ ”]
5. Given the set of feasible schedules identified in point 3 above and the set of feasible patterns for the week " $w+1$ " identified in point 4 , we perform a search in this schedule space to find feasible schedules for week “ $\mathrm{w}+1$ ”.

[The elimination of some of the patterns as a possible continuation after a specific pattern in week " $w$ " (as highlighted in point 4 ) reduces the number of possible schedules that can be generated from this smaller set of patterns in week " $w+1$ ". Consequently some of the 380 schedules that were feasible in week " $w$ " will not be feasible in week " $\mathrm{w}+1$ ". However, an important conclusion is that a set of feasible schedules for week " $w+1$ " is contained in the set of feasible schedules for week "w". This provides an upper limit on the extent of search that is needed to identify all feasible schedules in week " $w+1$ ". An example of a single feasible schedule for week " $w+1$ " is given in Table 5]

6. The process of scheduling personnel for subsequent weeks is implemented by repeating steps 4 and 5 above until the set of feasible patterns is empty of the planning horizon has been reached..

\section{Computational results}

Figure 3 illustrates the granulation of the problem space and the consequent reduction of the set of feasible patterns.

\section{Week 1}

Week 2

Week 3

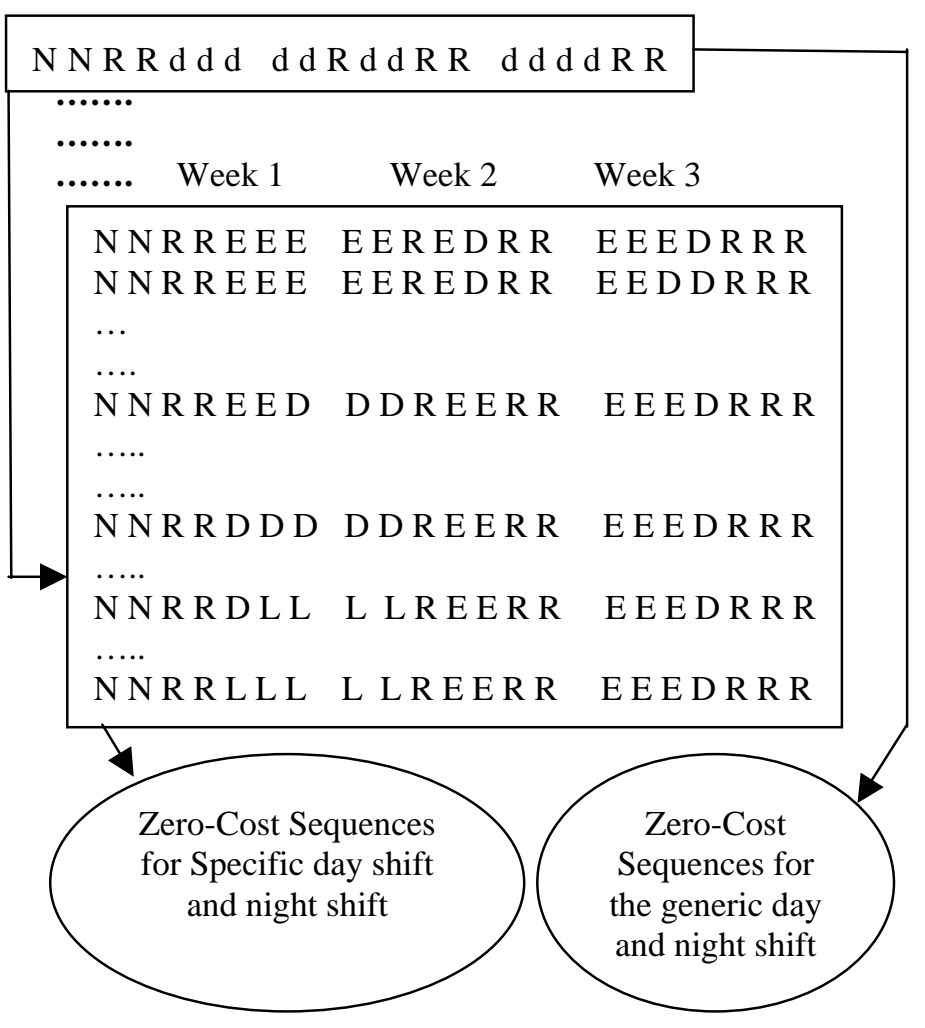

Figure 3: Offline preparation Process 
The constraints on the required personnel cover in the granulated problem space are represented in Table 3.

Table 3: Demand summarization

\begin{tabular}{|c|c|c|c|c|c|c|c|}
\hline \multicolumn{9}{|c|}{ Demand } \\
\hline & M & T & W & T & F & S & S \\
\hline $\mathrm{d}$ & 9 & 9 & 9 & 9 & 9 & 6 & 6 \\
\hline $\mathrm{N}$ & 1 & 1 & 1 & 1 & 1 & 1 & 1 \\
\hline
\end{tabular}

Table 4 shows the 26 feasible shift patterns which were identified for the sample problem of a ward in a Duch hospital. Table 5 shows a representative 2-week schedule for 15 nurses that satisfies the problem constraints (i.e. it is a zero-cost schedule)..

Table 4: 26 common patterns

\begin{tabular}{|c|c|}
\hline Pattern & Shift Sequence \\
\hline P1 & N N R R R d d \\
\hline $\mathrm{P} 2$ & N N R R d d d \\
\hline P3 & d d N N R R R \\
\hline $\mathrm{P} 4$ & R R R d N N N \\
\hline $\mathrm{P} 5$ & R R d d N N N \\
\hline P6 & d d R R N N N \\
\hline P7 & R d d R N N N \\
\hline P8 & d R R R N N N \\
\hline P9 & d R R d N N N \\
\hline $\mathrm{P} 10$ & R R d d R d d \\
\hline $\mathrm{P} 11$ & $\mathrm{R} \mathrm{d} \mathrm{d} \mathrm{R} \mathrm{R} \mathrm{d} \mathrm{d}$ \\
\hline P12 & $\mathrm{R} \mathrm{d} \mathrm{d} \mathrm{R} \mathrm{d} \mathrm{d} \mathrm{d}$ \\
\hline P13 & $\mathrm{R} \mathrm{d} \mathrm{d} \mathrm{d} \mathrm{R} \mathrm{d} \mathrm{d}$ \\
\hline $\mathrm{P} 14$ & $\mathrm{R} \mathrm{d} \mathrm{d} \mathrm{d} \mathrm{d} \mathrm{R} \mathrm{R}$ \\
\hline P15 & d R R R d d d \\
\hline $\mathrm{P} 16$ & $\mathrm{~d}$ R d d R d d \\
\hline $\mathrm{P} 17$ & d R d d d R R \\
\hline P18 & d d R R R d d \\
\hline P19 & d d R d d R R \\
\hline P20 & d d d d R R R \\
\hline P21 & R R R d d d d \\
\hline $\mathrm{P} 22$ & R R d d d d d \\
\hline P23 & $\mathrm{d} R \mathrm{R} \mathrm{d} \mathrm{d} \mathrm{d} \mathrm{d}$ \\
\hline P24 & $\mathrm{d} d \mathrm{R} R \mathrm{Rd} \mathrm{d}$ \\
\hline P25 & $\mathrm{dddddRR}$ \\
\hline P26 & $\mathrm{d} d \mathrm{~d} R \mathrm{R} \mathrm{d} \mathrm{d}$ \\
\hline
\end{tabular}

As a rough guideline of the efficiency of the proposed algorithm we can report the following computational times on $1.64 \mathrm{GHz}$ PC with 448MB RAM:

a)generation of subsets, $\mathbf{0 . 1 5 6} \mathbf{~ s e c}$

b) computation of 26 patterns, $\mathbf{0 . 2 4 2} \mathbf{~ s e c}$

c) generation of week 1patterns : $\mathbf{3 . 9 5 4}$ secs

d) generation of the first 5 week schedule: $\mathbf{3 6 0 0} \mathbf{~ s e c}$
Table 5: A two-week schedule for 15 nurses

\begin{tabular}{|c|c|c|c|c|}
\hline $\begin{array}{l}\text { Nurse } \\
\text { No. }\end{array}$ & $\begin{array}{l}\text { Week1 } \\
\text { Pattern }\end{array}$ & $\begin{array}{l}\text { Week2 } \\
\text { Pattern }\end{array}$ & $\begin{array}{l}\text { Week1 } \\
\text { Sequences }\end{array}$ & $\begin{array}{l}\text { Week2 } \\
\text { Sequences }\end{array}$ \\
\hline N1 & P1 & P12 & NNRRRdd & RddRddd \\
\hline N2 & P3 & P23 & ddNNRRR & dddRRdd \\
\hline N3 & P4 & P22 & RRRdNNN & RRddddd \\
\hline N4 & P10 & $\mathrm{P} 1$ & RRddRdd & NNRRRdd \\
\hline N5 & P11 & P15 & RddRRdd & dRRRddd \\
\hline N6 & P11 & P15 & RddRRdd & dRRRddd \\
\hline N7 & P12 & P17 & RddRddd & dRdddRR \\
\hline N8 & P15 & P5 & dRRRddd & RRddNNN \\
\hline N9 & P17 & P20 & dRdddRR & ddddRRR \\
\hline N10 & P17 & P3 & dRdddRR & ddNNRRR \\
\hline N11 & P19 & P14 & ddRddRR & RddddRR \\
\hline N12 & P19 & P14 & ddRddRR & RddddRR \\
\hline N13 & P25 & P19 & dddddRR & ddddRRR \\
\hline N14 & P25 & P19 & dddddRR & ddRddRR \\
\hline N15 & P25 & P20 & dddddRR & ddRddRR \\
\hline Total & & & 9999966 & 9999966 \\
\hline
\end{tabular}

\section{CONCLUSION}

Nurse rostering problem considered at the level of detailed time constraints and different types of dayshifts represents a very significant computational challenge. In this paper we propose an alternative granular formulation of the problem that reduces the size of the problem space and allows the replacement of the extended-time scheduling with the recursive application of a week-at-a-time scheduling process. The nested nature of sets of feasible schedules for consecutive weeks gives rise to a natural hierarchical algorithm for nurse rostering.

The study also shows the efficiency of identifying feasible patterns for subsequent weeks. Conceptually this approach can be generalized to non-zero cost pattern sets. Automating the nurse rostering problem can actually reduce the scheduling effort and time. It can also take care of all the constraints and give a quick evaluation of schedules. Information granulation was found to be very useful in providing a good quality of solutions by reducing the explorations of paths. This identification and extraction of good features used more explicitly to solve the problem and hence to reduce the search space.

\section{REFERENCES}

Adbennadher S. and Schlenker H.1999. "Nurse Scheduling using Constraint Logic Programming” $11^{\text {th }}$ Annual Conference on Innovative Applications of Artificial Intelligence, IAAI-99. 838-843.

Aickelin U. and Dowsland K.2003.” An Indirect Genetic Algorithm for a Nurse Scheduling Problem.” JORS. 31(5). 761-778.

Anthony W. 1995. "Scheduling, timetabling and rostering - a special relationship?.PATAT(Lecture Notes in Computer Science) . Springer. Volume 1153. 46-75. 
Bard J. and Purnomo H.W. 2005. "Preference Scheduling for Nurses using Column Generation”. EJOR. 164: 510-534.

Beddoe G. and Petrovic S. 2005. "Determining Feature Weights Using a Genetic Algorithm in a CaseBased Reasoning Approach to Personnel Rostering”. Accepted for publication in EJOR.

Beaumont N.1997. " Scheduling Staff using Mixed Integer Programming”. EJOR, 98: 473-484.

Berrada, I., Ferland, J., and Michelon, P.1996.” A MultiObjective Approach to Nurse Scheduling with both Hard and Soft Constraints". Socio-Economic Planning Science. vl. 30.183-193

Brucker, P., Rong Qu, Burke, E., and Post, G. 2005.“A Decomposition, Construction And Post-Processing Approach For Nurse Rostering” Multidisciplinary International Scheduling Conference. New York, USA.(Jul). 397-406.

Burke E., De Causmaecker P. and Vanden Berghe G.1998. “ A Hybrid Tabu Search Algorithm for the Nurse Rostering Problem”. Selected Papers from the 2nd Asia Pacific Conference on Simulated Evolution and Learning. LNAI 1585, 187-194.

Burke E., Cowling P., De Causmaecker P. and Vanden Berghe

G.2001.”A Memetic Approach to the Nurse Rostering Problem”. Applied Intelligence. 15: 119214.

Burke E., De Causmaecker P., Vanden Berghe G. and Van Landeghem H.2004. "The State of the Art of Nurse Rostering”. Journal of Scheduling. 7(6). 441-499.

Burke E., Curtois T., Post G., Qu R. and Veltman B. 2005. “A Hybrid Heuristic Ordering and Variable Neighbourhood Search for the Nurse Rostering Problem”. Technical Report NOTTCS-TR-20053.School of CSiT, University of Nottingham

Cheang B., Li H., Lim A. and Rodrigues B. 2003. "Nurse Rostering Problems - a Bibliographic Survey”. EJOR. 151.447-460.

Duenas, A., Mort, N., Reeves, C., and Petrovic, D. 2003. “ Handling Preferences Using Genetic Algorithms for the Nurse Scheduling Problem". Proc.of the 1st MISTA. vol.1.180-195.

Ernst A.T., Jiang H., Krishnamoorthy M. and Sier D.2004. “ Staff Scheduling and Rostering: A Review of Applications, Methods and Models”. EJOR. 153: 327.

Gendrau, M., Buzon, I., Lapierre, S., Sadr, J., and Soriano, P. A. 2003. " Tabu Search Heuristic to Generate Shift Schedules”. Proc. of the 1st MISTA. vol.2.526-528

Hadi W. Purnomo and Jonathan F. 2007.” Cyclic Preference Scheduling for Nurses Using Branch and Price" Bard Naval Research Logistic. Vol. 54 No. 2. 200220. Published online in Wiley InterScience (www.interscience.wiley.com).

Lukman D., May J. Shuman L. and Wolfe H.1991. "Knowledge-Based Schedule Formulation and Maintenance Under Uncertainty". Journal of the Society of Health Systems. 2: 42-64.

Meyer H auf'm Hofe H.2000.” Solving Rostering Tasks as Constraint Optimisation". In: Selected Papers from the $3^{\text {rd }}$ International Conference on Practice and Theory of Automated Timetabling (PATAT'00), LNCS 2079.280-297.

Miller, H.E. , Pierskalla, W.P. and Rath, G.J. 1976.”Nurse scheduling using mathematical programming". Oper Res.24(5).857-870.
Nonobe K. and Ibaraki T.1998.” A Tabu Search Approach to the Constraint Satisfaction Problem as a General Problem Solver”. EJOR.. 106: 599-623.

Özcan, E. 2005. "Memetic Algorithms for Nurse Rostering”. In 20th International Symposium on Computer and Information Sciences. Springer-Verlag. 482-492.

Pierskalla W. and Rath G.1976.” Nurse Scheduling using Mathematical Programming” .OR, 24(5): 857-870.

Sitompul D. and Randhawa S.1990.” Nurse Rostering Models:

A State-of-the-Art Review". Journal of the Society of Health Systems. 2: 62-72.

Randhawa, S.U. and Sitompul, D. 1993.”A heuristic-based computerized nurse scheduling system”. Comput Oper Res.20(8).837-844.

Warner M. 1976. ” Scheduling Nursing Personnel According to Nurse Preference: a Mathematical Programming Approach.”. OR.24: 842-856.

\section{AUTHOR BIOGRAPHIES}

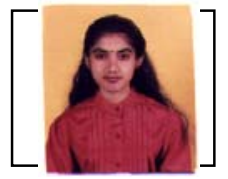

GEETHA BASKARAN was born in

Melaka, Malaysia. She completed her diploma and degree in Computer Science (Software Engineering) at University Putra Malaysia in the year 2000 and 2002

respectively. Two years later she enrolled in Master in Software Engineering (2004) in University of Malaya while working few years in the industry. She then joined University of Nottingham (Malaysia Campus) as a Assistant Professor in 2006 and currently pursuing her PhD studies here.

ANDRZEJ BARGIELA is Professor and Director of Computer Science at the University of Nottingham, Malaysia Campus. He is member of the Automated Scheduling and Planning research group in the School of Computer Science at the University of Nottingham. Since 1978 he has pursued research focused on processing of uncertainty in the context of modelling and simulation of various physical and engineering systems. His current research falls under the general heading of Computational Intelligence and involve mathematical modelling, information abstraction, parallel computing, artificial intelligence, fuzzy sets and neurocomputing.

DR RONG QU is a Lecturer in the School of Computer Science at the University of Nottingham. She gained her $\mathrm{PhD}$ in Computer Science from the University of Nottingham in 2002. Her main research areas include meta-heuristics, constraint programming, IP/ILP, case based reasoning methodologies and knowledge discovery techniques on scheduling, especially educational timetabling, healthcare personnel scheduling, network routing problems and graph colouring. In total she has more than 30 papers published or to appear at international journals and peer-reviewed international conferences. Dr $\mathrm{Qu}$ is also a guest editor for special issues at the journal of Memetic Computing and the Journal of Scheduling, and the program chair of several workshops and an IEEE symposium. 